\title{
Interaction of sex and practice distribution effects
}

\author{
ROBERT J. McCAFFREY and R. B. PAYNE \\ University of Georgia, Athens, Georgia 30602
}

\begin{abstract}
Recent studies have shown that sex dominance in psychomotor reminiscence undergoes reversal across pubescent years. On the assumption that dominance occurs because the sexes release reactive inhibition at different rates, it follows that, within a given developmental phase, the dominant sex should profit more from practice distribution. This implication was tested and confirmed in the present sample of 48 postpubescent subjects, and preliminary analyses of unreported data suggest that it will be confirmed in prepubescent samples as well. Thus, which sex gains the greater advantage from practice distribution depends upon developmental phase, and reactive inhibition theory seems able to handle such results regardless of developmental phase. Why these ontogenetic shifts occur remains obscure.
\end{abstract}

Numerous studies have shown that psychomotor reminiscence is far more complexly regulated than traditional accounts of it have suggested. Among the independent variables whose relevance has been most recently confirmed are sex, task, and developmental factors. For example, studies of young adults, using rotary pursuit and mirror tracking tasks, have shown that females reminisce more than males (Buxton \& Grant, 1939; Huang \& Payne, 1975; Payne \& Huang, 1977). However, studies of prepubescent children, using the same tasks, have shown that males reminisce more than females (Horn, 1975; Zegiob \& Payne, 1977). Given the replication of both findings, the conclusion seems inescapable that sex dominance in reminiscence on these tasks, and perhaps others, somehow undergoes reversal across pubescent years. This state of affairs is further complicated by the fact that no sex differences in reminiscence can be found on inverted alphabet printing tasks, and perhaps others, at either age level (Archer \& Bourne, 1956; Huang \& Payne, 1975; Zegiob $\&$ Payne, 1977). Thus, recovery from the decremental effects of massed practice seems to involve a complex interaction of sex, task, and developmental factors as well as the independent variables emphasized by Hull (1943).

Within the context of Hull's theory (1943), one can suppose that a sex difference in reminiscence occurs because the sexes release reactive inhibition $\left(I_{r}\right)$ at different rates. ${ }^{1}$ This appears to have been the case in Huang and Payne (1975), where female performance rebounded well out of proportion to its relative depression by massed practice. The argument is obviously circular, but it does have a transsituational implication for which empirical content can be

Requests for reprints should be sent to R. B. Payne, Department of Psychology, University of Georgia, Athens, Georgia 30602. adduced. Inasmuch as $I_{r}$ theory is also involved in Hull's interpretation of distributed practice effects, one might expect from the reminiscence data that females would profit more than males from practice distribution. Thus, a conventional spaced practice paradigm should produce an interaction of sex and distribution effects in which the sexes should perform about equally well under massed practice conditions, while females should perform better than males under distributed practice conditions. The present study was designed to examine this implication.

\section{METHOD}

\section{Subjects}

The experimental sample consisted of 48 subjects ( 24 male, 24 female) drawn from introductory classes in fulfillment of a course requirement. All were Caucasian, right-handed, and normally sighted. Their modal age was 18 years.

Task

The study employed a tracking task in which the subjects used mirror vision in order to keep a stylus on a small silver target as it moved clockwise at $1 \mathrm{rpm}$ through a narrow starshaped pathway (Payne \& Artley, 1972). Time on target to the nearest $.01 \mathrm{sec}$ was recorded for each minute of practice.

\section{RESULTS}

Average performance trends for the four combinations of sex and practice mode are shown in Figure 1. As expected, it is clear from these trends that distributed practice favored both sexes; but there is also a firm suggestion that females profited more from it than males, as predicted.

In order to provide a more stringent examination of experimental effects, the 960 scores $(48 \times 20)$ were subjected to a mixed-model variance analysis in which sexes and practice modes comprised the main between subjects effects and trials, the main within-subjects 


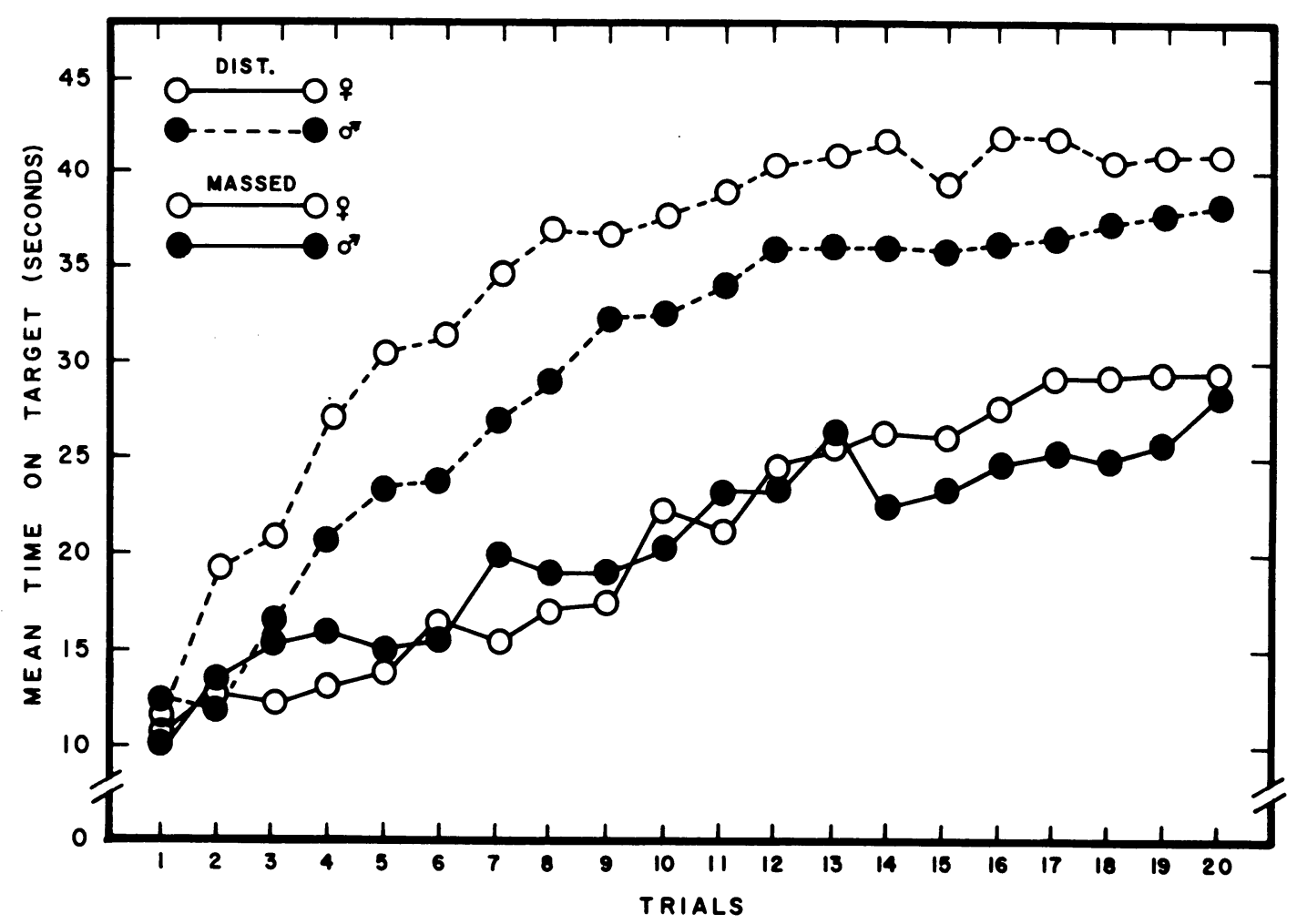

Figure 1. Performance trends under the four experimental conditions.

effect. The practice mode effect, of course, was highly significant $[F(1,44)=19.22, p<.001]$. The trial effect was also highly significant $[\mathrm{F}(19,836)=67.18, \mathrm{p}<.001]$. Also as expected, there was a significant interaction of Mode by Trial Effects $[F(19,836)=6.24, p<.001]$, in which mode differences first increased and then decreased as trials progressed. Although the expected interaction of Sex by Mode Effects was not significant $(F<1)$, there was a significant interaction of Sex by Mode by Trial Effects $[F(19,836)=3.88, p<.001]$. Remaining effects (sexes, trials by sexes) were not significant.

The foregoing analysis, coupled with inspection of Figure 1, seems to say that females profited more than males from practice distribution across some segments of the practice sequence, but not across all of them. In order to examine the simple effects involved in the significant triple interaction, the trial sequence, exclusive of Trial 1, was partitioned into roughly equal thirds (Trials 2-7, 8-13, 14-20), and mean performance for each subject was calculated for each third. Variancecovariance analyses, in which Trial 1 scores served as the covariate and segment means served as the dependent variable, were then conducted within each segment. Finally, adjusted sex mean differences within practice modes were examined for significance by $t$ tests in which the null hypothesis was evaluated against the one-sided alternative $\bar{y}_{\mathrm{a}}$ (female) $>\bar{y}_{\mathrm{a}}$ (male). Standard deviations of differences were derived from the adjusted error terms resulting from the variance-covariance analyses.
With regard to the first third of the practice sequence, $\bar{y}_{\mathrm{a}}$ (female) was significantly higher than $\bar{y}_{\mathrm{a}}$ (male) in the distributed mode $[t(43)=1.96, p<.05]$, while the sex means were essentially equal in the massed mode. During the second third, females persisted in their apparent superiority under the distributed mode, but the null hypothesis was not impugned $[\mathrm{t}(43)=1.51, \mathrm{p}>.05]$. The null hypothesis also prevailed in the final third for both practice modes.

In order to examine the significance of the alternative $\bar{y}_{\mathrm{a}}$ (female) $>\bar{y}_{\mathrm{a}}$ (male) across a broader segment of the practice sequence, consolidated means were computed for each subject of Trials 2-13. These were subjected to variance-covariance analyses as before, and the adjusted sex mean differences within modes were again tested for significance. Once again the $\bar{y}_{a}$ (female) was superior to the $\bar{y}_{\mathrm{a}}$ (male) $[\mathrm{t}(43)=1.85, \mathrm{p}<.05]$. The sex means were essentially equal in the massed mode. Thus, the prediction that $\bar{y}_{a}$ (female) $>\bar{y}_{a}$ (male) in the distributed mode, but not in the massed mode, was upheld within a practice segment that spanned essentially twothirds of the total practice sequence. It is obvious, of course, that the advantage realized by females from the distributed mode became smaller as trials progressed.

\section{DISCUSSION}

The credibility of the hypothesis that females reminisce more than males because they release $I_{r}$ more readily has obviously been enhanced by the present demonstration that they profit more than males from practice distribution, at least in the major 
portion of an extended practice sequence. Moreover, this interpretation is buttressed by the fact that the sexes were essentially equal under massed practice conditions, a fact which suggests that the sexes accumulated $\mathrm{I}_{\mathrm{r}}$ at comparable rates.

But what of the results of Horn (1975) and Zegiob and Payne (1977), in which prepubescent males reminisced more than females? The apparent reversal of sex dominance in reminiscence across pubescent years may be saying something vary basic about the role of ontogenetic factors in the regulation of this phenomenon. Whatever the cause of the apparent reversal, one can hypothesize, as in the prelude to the present paper, that prepubescent males reminisce more than their female counterparts because they release $I_{r}$ more readily. And, again, it follows from $I_{r}$ theory that prepubescent males should profit more than females from practice distribution. Resick and Payne (Note 1) have found at least marginal support for this expectation. If results continue to confirm it, we are faced with a developmental problem of extraordinary appeal.

\section{REFERENCE NOTE}

1. Resick, P. A., \& Payne, R. B. Sex and practice distribution effiects in children. In preparation.

\section{REFERENCES}

Archer, E. J., \& Bourne, L. E., JR. Inverted-alphabet printing as a function of intertrial rest and sex. Journal of Experimental Psychology, 1956, 52, 322-328.
Buxton, C. E., \& Grant, D. A. Retroaction and gains in motor learnng: II. Sex differences, and a further analysis of gains. Journal of Experimental Psychology, 1939, 25, 198-208.

HoRN, P. W. Pursuit rotor speed, sex differences, and reminiscence in young children. Journal of Psychology, 1975, 91, 81-85.

Huang, K. L.. \& Payne, R. B. Individual and sex differences in reminiscence. Memory \& Cognition, 1975, 3, 252-256.

Hull, C. L. Principles of behavior. New York: Appleton-CenturyCrofts, 1943.

Hull, C. L. The place of innate individual and species differences in a natural-science theory of behavior. Psychological Review, 1945, 52, 55-60.

Payne, R. B., \& ARTley, C. W. Facilitation of psychomotor learning by classically differentiated supplementary feedback cues. Journal of Motor Behavior, 1972, 4. 47-55.

Payne, R. B., \& Huang, C. L. Interaction of sex and task differences in reminiscence. Journal of Motor Behavior, 1977, 9, 29-32.

Zegiob, L., \& Payne, R. B. Reminiscence in children as a function of sex. Bulletin of the Psychonomic Society, 1977, 9. 173-175.

\section{NOTE}

1. This view, of course, is compatible with $I_{r}$ theory, since Hull (1945) provided for individual, group, and even species differences in terms of the empirical constants of equations purporting to describe behavioral laws.

(Received for publication July 6, 1977.) 